\title{
Jaundice as an Unusual Presentation of Kawasaki Disease: A Case Report
}

\author{
Ahmad Talebian ${ }^{1}$; Babak Soltani ${ }^{1, *} ;$ Mostafa Haji Rezaei $^{2}$ \\ ${ }^{1}$ Department of Pediatrics, Kashan University of Medical Sciences, Kashan, IR Iran \\ ${ }^{2}$ Trauma Research Center, Kashan University of Medical Sciences, Kashan, IR Iran \\ ${ }^{*}$ Corresponding author: Babak Soltani, Department of Pediatrics, Kashan University of Medical Sciences, Kashan, IR Iran. Tel: +98-03155575840, Fax: +98-03155548900, \\ E-mail: babak_soltani1969@yahoo.com
}

Received: February 2, 2015; Revised: February 21, 2015; Accepted: March 4, 2015

\begin{abstract}
Introduction: Atypical Kawasaki disease (KD) is a diagnostic dilemma. Acute acalculous cholestasis and liver involvement sometimes indicate atypical presentations of KD.

Case Presentation: We report a four-year-old boy with fever, abdominal pain, vomiting, jaundice and mild liver enlargement who gradually developed other signs of KD. He was treated by aspirin, intravenous immunoglobulin and finally methylprednisolone pulse therapy with no sequela.

Conclusions: KD should be kept in mind as one of the causes of childhood acalculous cholestasis, abdominal discomfort and prolonged fever.

Keywords: Jaundice; Kawasaki Disease; Liver; Cholestasis
\end{abstract}

\section{Introduction}

Kawasaki disease (KD) was reported about 40 years ago by Dr. Tomisaku Kawasaki. This disease is a systemic vasculitis of medium to small vessels, which mainly involves children less than five years of age (1).

$\mathrm{KD}$ is known also as mucocutaneous lymph node syndrome and is one of the most common causes of childhood vasculitis (2). Following a short period of nonspecific respiratory and gastrointestinal symptoms, a number of signs such as nonexudative conjunctivitis, erythema of lips and oral mucosa, rash, and cervical lymphadenitis appear. The mainstay diagnosis of KD is the presence of systemic inflammation, which shows itself by fever and mucocutaneous involvement. Fever is the most common clinical manifestation of KD. In many countries, KD has become the most common cause of childhood acquired heart disease, surpassing the acute rheumatic fever $(3,4)$. Gastrointestinal (GI) involvement does not belong to the classic criteria of KD and there is no specific and definitive diagnosis for this disease. Fifteen to forty five percent of children show atypical presentations (5). We presented a case of atypical KD which was masqueraded with jaundice.

\section{Case Presentation}

A previously healthy four-year-old boy was visited in the pediatric clinic of our center with fever since a day before. On physical exam, generalized maculopapular rashes, pharyngeal erythema and strawberry tongue were detected. There was no recent history of medica- tions. With the diagnosis of scarlet fever, a dose of intramascular penicillin 6:3:3 was prescribed and the patient was discharged. Two days later, he was hospitalized in our department due to persistent vomiting and abdominal pain. At the admission, physical examination revealed high grade fever, bilateral $1.5 \times 1.5 \mathrm{~cm}$ submandibular lymphadenopathies and strawberry tongue, but the rashes were eliminated. Sclera was icteric. Abdomen was not rigid but there was right upper quadrant (RUQ) tenderness, mild hepatomegaly with liver span of $8 \mathrm{~cm}$ that was palpated $3 \mathrm{~cm}$ below the right costal margin.

Biological evaluations showed leukocytosis of 17,500/ $\mathrm{mm}^{3}$ with neutrophil predominance (90\%), anemia with hemoglobin $10 \mathrm{~g} / \mathrm{dL}(11-15 \mathrm{~g} / \mathrm{dL})$, thrombocytosis (494,000/ $\mathrm{mm}^{3}$ ), elevated erythrocyte sedimentation rate (ESR) (64 $\mathrm{mm}$ in the first hour), high $\mathrm{C}$ reactive protein (CRP) level $(18 \mathrm{mg} / \mathrm{dL})$ and hypoalbuminemia $(2 \mathrm{~g} / \mathrm{dL})$. Liver function tests indicated hepatic involvement and particularly signs of cholestasis: mild increase of total $(5.2 \mathrm{mg} / \mathrm{dL})$ and direct (4.3 mg/dL) serum bilirubin, ALT $260 \mathrm{U} / \mathrm{L}$ (5 - $45 \mathrm{U} / \mathrm{L})$, AST $159 \mathrm{U} / \mathrm{L}(15$ - $55 \mathrm{U} / \mathrm{L})$, alkaline phosphatase $530 \mathrm{U} / \mathrm{L}$ (145 $420 \mathrm{U} / \mathrm{L}$ ), and gamma-glutamyl transpeptidase $270 \mathrm{U} / \mathrm{L}$ (5 - $32 \mathrm{U} / \mathrm{L}$ ). Amylase and lipase were within normal ranges. The ultrasonography of gallbladder and hepatobiliary ducts was reported normal with mild hepatomegaly. The serological tests for viral hepatitis and leptospirosis were negative. Wright, Coombs wright and BACTEC blood culture for salmonellosis were negative. Search for collagen vascular disorders and autoimmune hepatitis was insignificant. Serum alpha-1 antitrypsin was sufficient. Serum

Copyright (C) 2015, Pediartric Infections Research Center. This is an open-access article distributed under the terms of the Creative Commons Attribution-NonCommercial 4.0 International License (http://creativecommons.org/licenses/by-nc/4.0/) which permits copy and redistribute the material just in noncommercial usages, provided the original work is properly cited. 
ceruloplasmin and 24-hour urine copper levels were normal. Ophthalmologic consult for uveitis and KayserFleischer rings was negligible. It is necessary to mention that diagnostic tools such as serology, cultures and etc. should not delay the treatment of KD.

On the sixth day of admission, fever continued and bilateral nonpurulent conjunctivitis, cracking of lips, palmar and plantar erythema were added and according to the history of bilateral submandibular lymphadenopathy, strawberry tongue and the rashes, KD was diagnosed. High-dose (2 $\mathrm{g} / \mathrm{kg}$ ) intravenous immunoglobulin (IVIG) and aspirin (ASA) $100 \mathrm{mg} / \mathrm{kg} /$ day were started, but due to the continuation of fever after 48 hours, another dose of IVIG was prescribed and finally with persistence of fever for further two days, a pulse of methylprednisolone (30 $\mathrm{mg} / \mathrm{kg}$ infusion) was begun for a day, after which his fever stopped and the clinical manifestations improved. After four days of discontinuation of fever, high-dose ASA was changed to daily low-dose ASA for a total duration of eight weeks. In the second week of his illness, desquamation of fingers and elbows occurred and the platelet count rose to $1,132,000 / \mathrm{mm}^{3}$. Serial echocardiography during admission, two and six weeks later were normal. After the three-month follow-up of our patient, hepatomegaly disappeared and liver enzymes and bilirubin levels returned to the normal ranges.

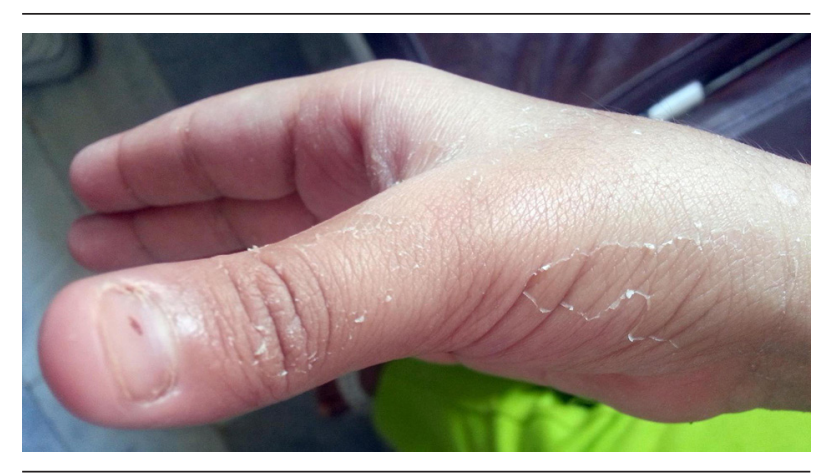

Figure 1. Desquamation of Kawasaki Disease

\section{Discussion}

Kawasaki disease is the second most common cause of childhood vasculitis and its diagnosis is mainly based on clinical manifestations. In the absence of specific diagnostic test for KD, a clinical criterion was offered by the Japanese Ministry of Health, which was approved by the american heart association (6).

The presence of gastrointestinal complaints such as diarrhea, vomiting and abdominal pain are observed in one third of patients, but hepatitis is an uncommon finding in $\mathrm{KD}$ (7). One of the atypical presentations of KD is hepatic involvement and the most important manifestations include jaundice and gallbladder hydrops (8). On the other hand, approximately $30 \%$ of children with KD manifest mild elevations of transaminase levels (9). Occasionally, atypical KD can mimic acute abdomen, and invasive surgical interventions may be used. According to a cohort study by Zulian et al. (7) on 219 children with KD, 10 (4.6\%) cases presented acute abdominal complaints. Nine of them had clinical manifestations of atypical KD at the onset such as severe abdominal pain, hepatomegaly, vomiting and jaundice. Laparotomy was performed in five patients, three had percutaneous transhepatic biliary drainage and gastrointestinal endoscopy was conducted in one case. Five patients had gallbladder hydrops, three had paralytic ileus and one had hemorrhagic duodenitis postoperatively. All of them recovered completely, but 50\% developed coronary artery aneurysms in spite of early intravenous immunoglobulin. Therefore, KD should be kept in mind as the differential diagnosis of acute surgical abdomen.

Seve et al. (10) evaluated 57 cases of adult KD and compared their clinical manifestations with reported children with KD in the literature. They concluded that cheilitis, thrombocytosis and meningitis were more likely to occur in children, whereas adenopathy, liver abnormality and arthralgia were seen more commonly in adults.

Taddio et al. (11) reviewed 22 cases who were hospitalized due to febrile acalculous cholestatic jaundice in two tertiary pediatric centers. Viral infection was the most common cause followed by KD (21\%) and drug-induced liver damage (16\%). They also ruled out autoimmune hepatitis and Wilson's disease in all patients. Although we ruled out Wilson's disease and serum alpha-1 antitrypsin deficiency in our patient, they were at the end list of differential diagnoses of acute febrile acalculous cholestatic jaundice in a child and it should be kept in mind that the diagnosis of typical and incomplete KD is clinical and early treatment should not be postponed by time-consuming diagnostic modalities such as serological tests, cultures and miscellaneous. We did not find any studies that measured alpha-1 antitrypsin in similar cases.

Karpathios et al. (12) reported a seven-year-old boy of KD with jaundice (total bilirubin of $7.45 \mathrm{mg} / \mathrm{dL}$ and direct component of 4.86). Due to severity of jaundice, he was evaluated genotypically for Wilson's disease and Gilbert syndrome and finally Gilbert syndrome was documented. They considered the elevated bilirubin as a risk factor of coronary artery abnormalities. Indeed, it seems that the atypical features of KD predict a higher risk of developing coronary aneurysm; so, pediatricians should be vigilant for these diagnoses (13).

Gordon and coworkers reported a case of acute hepatitis in a three-year-old child who was mildly icteric at presentation and the liver function tests were impaired. All viral hepatic markers except for Epstein-Barr virus were negative. The patient was improved by intravenous immunoglobulin injection without any cardiac complications (14). Our patient had no evidence of viral and autoimmune hepatitis and other causes of hepatitis were not found. Furthermore, because of normal reticulocyte count (0.5\%) and direct hyperbilirubinemia, hemolytic anemia was ruled out in our case. 
A 10-year-old boy was presented by Dr. Chen with marked impairment of hepatobiliary tract, jaundice and prolonged fever. He did not have any conjunctivitis until the eighth day of admission. Desquamative lesions of extremities and strawberry tongue began on the 13th day of hospitalization. In spite of standard therapy of KD, multiple coronary artery aneurysms were ensued (8).

Our patient and Gordon's case were less than five years of age and were treated without any consequences, but Chen's patient was 10 years of age and cardiac complication was resulted. In Gordon's (15) and our cases, the documented clinical criteria of KD appeared earlier; so, prompt diagnosis and treatment were made sooner and no sequela remained. On the opposite, Chen's patient showed the diagnostic criteria later and cardiac complication was resulted (8). Early diagnosis and treatment of KD is imperative for any physicians to prevent its complications (8).

Pannaraj indicated that $52 \%$ of general practitioners and $25 \%$ of infectious diseases specialists did not consider $\mathrm{KD}$ as the differential diagnosis of children less than six months or more than eight years of ages (14). The gastrointestinal manifestations of KD are nonspecific. Severe abdominal pain with diarrhea was reported in $20 \%$ and obstructive jaundice associated with hepatic dysfunction due to gallbladder hydrops in $13 \%$ of cases (16). Gallbladder hydrops is identified by right upper quadrant tenderness and abdominal mass which is sometimes asymptomatic and often improved without any surgical intervention (16). Histologically, sterile inflammatory perivascular infiltration and occasionally acute intrahepatic cholangitis associated with epithelial cell necrosis of biliary ducts are observed (17). In our patient, mild hepatomegaly, hepatitis and acute acalculous cholestasis with normal biliary ducts were presented. The pathogenesis of abdominal signs and symptoms in KD is due to small and moderate sized vasculitis, serositis, biliary ductal compression by adjacent mesenteric lymph nodes and gallbladder hydrops (17). Sometimes the differentiation between Scarlet fever and KD is difficult and it was compatible with our case; so, penicillin had no effect on him. Other causes of fever and jaundice including brucellosis, typhoid fever, leptospirosis, viral infections and collagen vascular diseases were negative in our case. Falcini et al. (18) supported that in any children with acute febrile acalculous cholestasis of unknown cause, KD must be suspected and prompt therapy initiated for prevention of coronary involvement.

In conclusion, there was no evidence of other causes of hepatitis and jaundice in our patient and most possibly, they were accounted as the atypical presentations of KD. According to the rarity of these manifestations and the importance of early diagnosis of KD, all of the physicians especially pediatricians should be vigilant against atypical presentations of this disease.

\section{Authors' Contributions}

Ahmad Talebian referred the patient to pediatric infectious service and supervised manuscript writing. Babak Soltani diagnosed the disease and wrote the case report. Mostafa Haji Rezaei searched the updated references and assisted in manuscript drafting.

\section{References}

1. Uehara R, Belay ED. Epidemiology of Kawasaki disease in Asia, Europe, and the United States. J Epidemiol. 2012;22(2):79-85.

2. Rowley AH, Shulman ST. Kawasaki Syndrome. Pediatr Clin North Am. 1999;46(2):313-29.

3. Baker AL, Lu M, Minich LL, Atz AM, Klein GL, Korsin R, et al. Associated symptoms in the ten days before diagnosis of Kawasaki disease. J Pediatr. 2009;154(4):592-595 e2.

4. Ozdemir H, Ciftci E, Tapisiz A, Ince E, Tutar E, Atalay S, et al. Clinical and epidemiological characteristics of children with Kawasaki disease in Turkey.J Trop Pediatr. 2010;56(4):260-2.

5. Singh S, Kawasaki T. Kawasaki disease - an Indian perspective. Indian Pediatr. 2009;46(7):563-71.

6. Newburger JW, Takahashi M, Gerber MA, Gewitz MH, Tani LY, Burns JC, et al. Diagnosis, treatment, and long-term management of Kawasaki disease: a statement for health professionals from the Committee on Rheumatic Fever, Endocarditis, and Kawasaki Disease, Council on Cardiovascular Disease in the Young, American Heart Association. Pediatrics. 2004;114(6):1708-33.

7. Zulian F, Falcini F, Zancan L, Martini G, Secchieri S, Luzzatto C, et al. Acute surgical abdomen as presenting manifestation of Kawasaki disease. J Pediatr. 2003;142(6):731-5.

8. Chen WT, Huang SR, Wang JK. Kawasaki disease presenting with hepatitis and prolonged fever: report of one case. Acta Paediatr Taiwan. 2003;44(3):174-6.

9. Burns JC, Mason WH, Glode MP, Shulman ST, Melish ME, Meissner $C$, et al. Clinical and epidemiologic characteristics of patients referred for evaluation of possible Kawasaki disease. United States Multicenter Kawasaki Disease Study Group. J Pediatr. 1991;118(5):680-6.

10. Seve P, Stankovic K, Smail A, Durand DV, Marchand G, Broussolle C. Adult Kawasaki disease: report of two cases and literature review. Semin Arthritis Rheum. 2005;34(6):785-92.

11. Taddio A, Pellegrin MC, Centenari C, Filippeschi IP, Ventura A, Maggiore G. Acute febrile cholestatic jaundice in children: keep in mind Kawasaki disease. J Pediatr Gastroenterol Nutr. 2012;55(4):380-3.

12. Karpathios T, Moustaki M, Yiallouros P, Sharifi F, Attilakos A, Papadopoulou A, et al. Severe jaundice in two children with Kawasaki disease: a possible association with Gilbert syndrome. J Korean Med Sci. 2012;27(1):101-3.

13. Valentini P, Ausili E, Schiavino A, Angelone DF, Focarelli B, De Rosa $\mathrm{G}$, et al. Acute cholestasis: atypical onset of Kawasaki disease. Dig Liver Dis. 2008;40(7):582-4.

14. Pannaraj PS, Turner CL, Bastian JF, Burns JC. Failure to diagnose Kawasaki disease at the extremes of the pediatric age range. Pediatr Infect Dis J. 2004;23(8):789-91.

15. Gordon MM, Silverman ED, Kim JH, Huber AM, Furuya K. Acute severe hepatitis with coagulopathy: An unusual presentation of Kawasaki syndrome in association with Epstein-Barr virus. Paediatr Child Health. 2001;6(9):627-30.

16. Suddleson EA, Reid B, Woolley MM, Takahashi M. Hydrops of the gallbladder associated with Kawasaki syndrome. J Pediatr Surg. 1987;22(10):956-9.

17. Liebmann LI, Mikelic V, Joh MM, Wilson FM. Hydrops of the gallbladder in an adult with Kawasaki disease. JAMA. 1982;247(6):827-9.

18. Falcini F, Resti M, Azzari C, Simonini G, Veltroni M, Lionetti P. Acute febrile cholestasis as an inaugural manifestation of Kawasaki's disease. Clin Exp Rheumatol. 2000;18(6):779-80. 